Portland State University

PDXScholar

Mechanical and Materials Engineering Faculty

Publications and Presentations

2-12-2016

\title{
Structure Functions, Scaling Exponents and Intermittency in the Wake of a Wind Turbine Array
}

\author{
Naseem Ali \\ Portland State University \\ Aleksandr Sergeyevich Aseyev \\ Portland State University \\ Raúl Bayoán Cal \\ Portland State University
}

Follow this and additional works at: https://pdxscholar.library.pdx.edu/mengin_fac

Part of the Materials Science and Engineering Commons, and the Mechanical Engineering Commons Let us know how access to this document benefits you.

\section{Citation Details}

Ali, N., Aseyev, A. S., \& Cal, R. B. (2016). Structure functions, scaling exponents and intermittency in the wake of a wind turbine array. Journal of Renewable and Sustainable Energy, 8(1), 013304.

This Article is brought to you for free and open access. It has been accepted for inclusion in Mechanical and Materials Engineering Faculty Publications and Presentations by an authorized administrator of PDXScholar. Please contact us if we can make this document more accessible: pdxscholar@pdx.edu. 


\section{A Journal of Renewable and Sustainable Energy}

Structure functions, scaling exponents and intermittency in the wake of a wind turbine array

Naseem Ali, Aleksandr S. Aseyev, and Raúl Bayoán Cal

Citation: Journal of Renewable and Sustainable Energy 8, 013304 (2016); doi: 10.1063/1.4941782

View online: http://dx.doi.org/10.1063/1.4941782

View Table of Contents: http://scitation.aip.org/content/aip/journal/jrse/8/1?ver=pdfcov

Published by the AIP Publishing

\section{Articles you may be interested in}

On the statistics of wind turbine wake meandering: An experimental investigation

Phys. Fluids 27, 075103 (2015); 10.1063/1.4923334

Anisotropy of the Reynolds stress tensor in the wakes of wind turbine arrays in Cartesian arrangements with counter-rotating rotors

Phys. Fluids 27, 015102 (2015); 10.1063/1.4903968

The effects of mean atmospheric forcings of the stable atmospheric boundary layer on wind turbine wake J. Renewable Sustainable Energy 7, 013124 (2015); 10.1063/1.4907687

Unsteady vortex lattice method coupled with a linear aeroelastic model for horizontal axis wind turbine J. Renewable Sustainable Energy 6, 042006 (2014); 10.1063/1.4890830

The interaction of helical tip and root vortices in a wind turbine wake

Phys. Fluids 25, 117102 (2013); 10.1063/1.4824734

\section{AlP $\mid$ APL Photonics}

APL Photonics is pleased to announce Benjamin Eggleton as its Editor-in-Chief

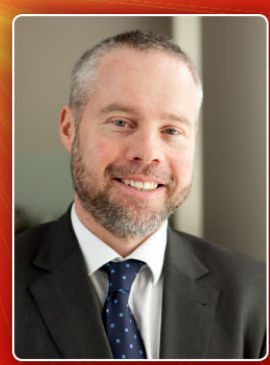




\title{
Structure functions, scaling exponents and intermittency in the wake of a wind turbine array
}

\author{
Naseem Ali, Aleksandr S. Aseyev, and Raúl Bayoán Cal \\ Department of Mechanical and Materials Engineering, Portland State University, Portland, \\ Oregon 97207, USA
}

(Received 5 May 2015; accepted 29 January 2016; published online 12 February 2016)

\begin{abstract}
Hot-wire measurements obtained in a $3 \times 3$ wind turbine array boundary layer are utilized to analyze high order structure functions, intermittency effects as well as the probability density functions of velocity increments at different scales within the energy cascade. The intermittency exponent is found to be greater in the far-wake region in comparison with the near-wake. At hub height, the intermittency exponent is found to be null. Extended self-similarity scaling exponents of the second, fourth, and fifth order structure functions remain relatively constant as a function of height in the far-wake; whereas in the near-wake, these are highly affected by the passage of the rotor where tip vortices reside, thus showing a dependence on physical location. When comparing with proposed models, these generally overpredict the structure functions in the far-wake region. The probability density function distributions in the far-wake region display wider tails compared to the near-wake region, and the constant skewness hypothesis based on the local isotropy is disrupted in the wake. (C) 2016 AIP Publishing LLC. [http://dx.doi.org/10.1063/1.4941782]
\end{abstract}

\section{INTRODUCTION}

Wind energy has become an important source in renewable energy due to an increasing demand for clean energy according to G. W. E. Council. ${ }^{1}$ Zhang et $a .^{2}$ found that characterizing the wake of the wind turbine array and its interaction with the atmospheric boundary layer is crucial, thus leading to a significant increase in power production. The wake of the wind turbine array tends to be rather complex as the passage of the blades, cumulative effects of wake to wake interactions, and the presence of a boundary layer produce different structures at different streamwise and wall-normal distances as stated in Ref. 3. Extensive studies carried out experimentally and numerically deal with characterizing the wake through analysis of mean and turbulence quantities with the goal of obtaining maximum power production, see Refs. 4-6. A common description of the statistical behavior of turbulent wakes is often achieved via the structure functions, thus possessing the ability to identify the flow structure and particularly determine isotropy levels through a statistical description. Chamecki and Dias ${ }^{7}$ used second and third order structure functions to investigate isotropic behavior in a surface-layer turbulent flow. Furthermore, Tatarskii ${ }^{8}$ used the second order structure function to identify the inertial subrange of the energy cascade.

The structure function $B(R)$ of order $p$ is defined in terms of the moments of the velocity increment as

$$
B(R)^{p}=\left\langle(u(x+R)-u(x))^{p}\right\rangle=\left\langle\delta_{R}(u)^{p}\right\rangle,
$$

where $\langle\ldots\rangle$ represents the ensemble average, $u$ is the streamwise velocity component at location $x$, and $R$ is a spatial separation distance between two points, which indeed quantifies a particular scale of interest. Commonly used scales to describe the flow are: the integral length scale, Taylor microscale and Kolmogorov microscale defined by $L=\int \rho_{\tau} d \tau, \lambda^{2}=\frac{\left\langle u(t)^{2}\right\rangle}{\left\langle\left(\frac{\partial u(t)}{\partial t}\right)^{2}\right\rangle}, \eta=\left(\frac{v^{3}}{\langle\varepsilon\rangle}\right)^{\frac{1}{4}}$, where $\rho_{\tau}, v, t, \tau, \varepsilon$ are autocorrelation coefficient, kinematic viscosity, time, time lag and 
dissipation, respectively. Local isotropy and the Taylor frozen field hypothesis are employed to estimate the dissipation as $\varepsilon=15 v\left\langle\left(\frac{\partial u}{\partial x}\right)^{2}\right\rangle$.

Kolmogorov, ${ }^{9}$ hereafter K41, introduced a similarity theory for homogenous and isotropic turbulent flows, which states that the mean dissipation energy, $\varepsilon$, is scale invariant and the structure function is given by

$$
B(R)^{p} \sim\langle\varepsilon\rangle^{\frac{p}{3}} R^{\frac{p}{3}}
$$

Higher order statistics display discrepancies when comparing the evaluation as proposed by K41 to experimental data as intermittency is taken into account, where a significant deviation from the mean is found in the dissipation as shown in Refs. 10 and 11. Phenomenological models and modification to the similarity theory, $\mathrm{K} 41$, have been proposed, where intermittency effects are addressed. Kolmogorov ${ }^{12}$ (K62) refined the previous similarity theory and presented a log-normal distribution for scale dependence within the dissipation range as

$$
B(R)^{p} \sim\left\langle\varepsilon_{R}\right\rangle^{\frac{p}{3}} R^{\frac{p}{3}} \sim R^{\xi_{p}} .
$$

Vassilicos ${ }^{13}$ highlighted that intermittency phenomenon can be recognized via the scaling exponent of appropriate moments, which associates the separation scales between two neighboring points. The scaling exponent, $\xi_{p}$, is described as

$$
\xi_{p}=\frac{p}{3}+\frac{1}{18} \mu p(3-p)
$$

where $\mu$ is the intermittency exponent, which characterizes the intermittency of the fluctuation of energy dissipation. Frisch et al. ${ }^{14}$ introduced the Beta-model, which is dependent on the energy cascade and concentrates on the transmission of nonlinearity in the inertial subrange. The scaling exponent of the Beta model can be expressed as

$$
\xi_{p}=\frac{p}{3}+\frac{1}{3}(3-H)(3-p)
$$

where $H$ is the Hausdroff dimension, otherwise known as the self-similarity dimension of the dissipative structures and defined as $H=3-\mu$, see Ref. 15. Combining $H$ with Eq. (5), the scaling exponent expression becomes

$$
\xi_{p}=\frac{p}{3}+\frac{1}{3} \mu(3-p)
$$

A low Reynolds number produces a short inertial subrange, thus making the scaling behavior either difficult to distinguish or nonexistent. To overcome this obstacle, Benzi et al. found that the scaling properties can be expanded up to the dissipative range as stated in Ref. 16. Furthermore, Benzi et al. ${ }^{17}$ introduced extended self-similarity (ESS) which also yields the scaling exponent and has been applied to high- and low-Reynolds number flows, e.g., Refs. 18 and 19 , as well as homogeneous and non-homogeneous flows, e.g., Refs. 20 and 21 . The scaling exponents $\xi_{p}$ of order $p$ are obtained via plotting the $\log \left(B_{R}{ }^{p}\right)$ against the $\log \left(B_{R}{ }^{3}\right)$, where the slope of the resulting line corresponds to $\xi_{p}$. Thus, it represents the relationship between the $B_{R}{ }^{p}$ and $B_{R}{ }^{3}$.

She and Leveque ${ }^{22}(\mathrm{SL})$, presented a model estimating the scaling exponent through its dependence on the hierarchical structure of energy dissipation as

$$
\xi_{p}=\frac{p}{9}+2\left(1-\left(\frac{2}{3}\right)^{\frac{p}{3}}\right) .
$$


Dubrulle $^{23}$ presented a novel interpretation of SL model using log-Poisson statistics. This model showed the scale covariance extending from the integral length scale to the dissipative scale, thus playing a central role in intermittency generation. Babiano et al. ${ }^{18}$ proposed a new model depending on SL and Dubrulle models for non-homogeneous and/or non-stationary turbulent flow. The maximum amplitude of the intermittency and the degree of inhomogeneity are linked via this approach.

Intermittency in turbulence has been investigated for different types of flows such as atmospheric boundary layer flow, e.g., Ref. 24, wake flow around a cylinder, e.g., Ref. 21, turbulent jet, e.g., Ref. 25, and direct numerical simulation (DNS) homogenous flow, e.g., Ref. 26. Ditlevsen and Mogensen ${ }^{27}$ investigated intermittency in a shell model and showed increased intermittency as the Kolmogorov scale is reached. Furthermore, energy traveling through the inertial range increases when the small scales are approached. Milan et al. ${ }^{28}$ presented a model of a conversion process between the wind speed and electrical power via multi-fractal statistics. Velocity and power increments over time scales were quantified, where the latter exhibited relatively higher intermittency as a result of the large fluctuations with frequent wind gusts. Consequently, fluctuations of electrical power in the grid were manifested.

Intermittent flow generates unfavorable influences such as variable dynamic loading and fatigue on the blades as well as the gearbox of the wind turbines. Mücke et al. ${ }^{29}$ demonstrated that the variable loads accelerate the strain and reduce the life of the turbine components. Therefore, intermittency analysis should be taken into account to minimize the design failure engendered from underestimated fatigue loads. Furthermore, intermittency analysis is useful in determining the spacing between the turbines in the wind farm in order to harvest an optimal power and achieve increased stability in the grid.

The present work analyzes the scaling exponents and intermittency exponents using extended self-similarity in the wake of a wind turbine array obtained experimentally by way of hot-wire anemometry. Scaling exponents acquired using ESS are compared with the K41, K62, SL, and Beta models. The probability density function of the velocity increment, flatness, and skewness is also investigated to show the degree of existing intermittency. Analysis of highorder structure functions can allow for predicting intermittency in the wind farm.

\section{EXPERIMENTAL SETUP}

Hot-wire data used in this study were gathered in the wake of a $3 \times 3$ wind turbine array and acquired from the Corrsin wind tunnel at the Johns Hopkins University. The test section is $10 \mathrm{~m}$ long, $0.9 \mathrm{~m}$ high, and $1.2 \mathrm{~m}$ wide. The test section and inflow conditioning elements used are described herein and shown in Fig. 1. An active grid consisting of seven vertical and five horizontal rotating aluminum shafts with winglets evenly spaced is used to generate high inflow turbulence. An atmospheric-like boundary layer is created with the use of nine strakes located at $1.07 \mathrm{~m}$ downstream of the active grid and distributed evenly from the sidewalls of the test section. Surface roughness composed of sandpaper is used in order to further condition the inflow. The construction of the 3-bladed wind turbine rotor requires a $0.48 \mathrm{~mm}$ thick steel plate, which is laser-cut and twisted $15^{\circ}$ at the root and $10^{\circ}$ at the tip using a die-press for repeatability. The induction factor equals to 0.087 . Using the ideal stream-tube analysis, the thrust and

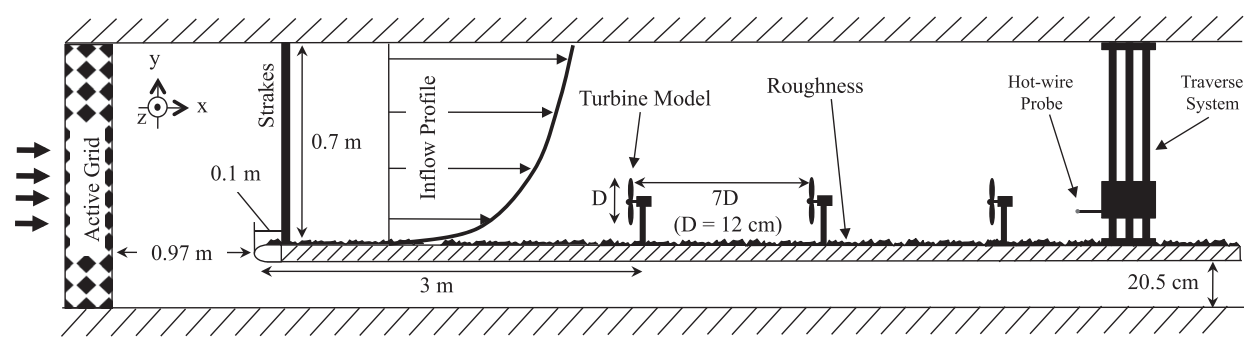

FIG. 1. Experimental setup for the wind turbine array boundary layer. 
power coefficients are approximately 0.32 and 0.29 , respectively. As seen in Fig. 2, the diameter of the rotor, $D$, and height of the mast are $0.12 \mathrm{~m}$.

The array is spaced at $7 D$ in the streamwise direction, $x$, and $3 D$ in the spanwise direction, $z$. The array is positioned $3 \mathrm{~m}$ downstream of the leading edge and $1.5 \mathrm{D}$ from the sidewalls. Two downstream locations at the centerline past the wind turbine array are considered to quantify the intermittency events in the near- and far-wake, namely, $1 D$ and $5 D$. Each profile consists of 21 measurements at $10 \mathrm{~mm}$ increments along the wall-normal direction, $y$, starting at $5 \mathrm{~mm}$ from the wall. The data are collected using an $\mathrm{x}$-wire probe with a sampling frequency of $40 \mathrm{kHz}$ for $100 \mathrm{~s}$ at each location.

The reference mean velocity of the wind tunnel is maintained constant throughout the experiment at $9.4 \mathrm{~m} \mathrm{~s}^{-1}$ and is measured using a pitot tube at $0.32 \mathrm{~m}$ upstream of the active grid as well as at $0.22 \mathrm{~m}$ downstream of the secondary contraction of the wind tunnel. To ensure the uniformity of the velocity profile in the spanwise direction, the mean velocity and Reynolds stresses are examined at fixed streamwise and wall-normal locations, and traversed from $z=-0.24 \mathrm{~m}$ to $0.24 \mathrm{~m}$ with an increment of $0.02 \mathrm{~m}$. The results show a reasonable homogeneity of the flow in the spanwise direction with a maximum deviation of mean velocity and Reynolds stress equal to $0.36 \mathrm{~m} \mathrm{~s}^{-1}$ and $1 \%$, respectively.

At the inflow, streamwise spectra are determined and compared with the Kolmogorov power law (see Fig. 8 in Ref. 30). There is a significant agreement with $-5 / 3$ line over a large range of scales; thus, energy spectra are used to recognize the inertial subrange of the energy cascade. ${ }^{31}$ Extensive details on the experimental setup, hot-wire anemometer, and flow characterization can be found in Refs. 30-32.

\section{RESULTS}

The sixth order of the ESS scaling exponents is used to calculate the intermittency exponent, $\mu=2-\xi_{6}$, as reported by Böttcher et al. ${ }^{33}$ The intermittency exponents, $\mu$, are presented as a function of wall-normal distance, $y$, normalized with the rotor diameter for downstream positions of $1 D$ and $5 D$, as observed in Fig. 3. The intermittency exponent in the far-wake monotonically increases with increasing wall-normal distance with the exception of very near the wall as well as hub height $(y / D=1)$, where local maxima are found to be 0.5 and 0.6 , respectively. This behavior is different from that observed in the near-wake, where values for $\mu$ are lower in magnitude, ranging from null to 0.4. The effect of the passage of the rotor is unequivocally present, thus generating tip vortices, where strong gradients of the exponent occur at the top tip $(y / D=1.5)$, bottom tip $(y / D=0.5)$, and hub height $(y / D=1)$. At the bottom tip and hub height locations, the exponent is null indicating that the intermittency is well suppressed for the higher order statistics. Kuznetsov et al. ${ }^{34}$ classified intermittency as internal or external. Dissipative scales are responsible to create the internal intermittency
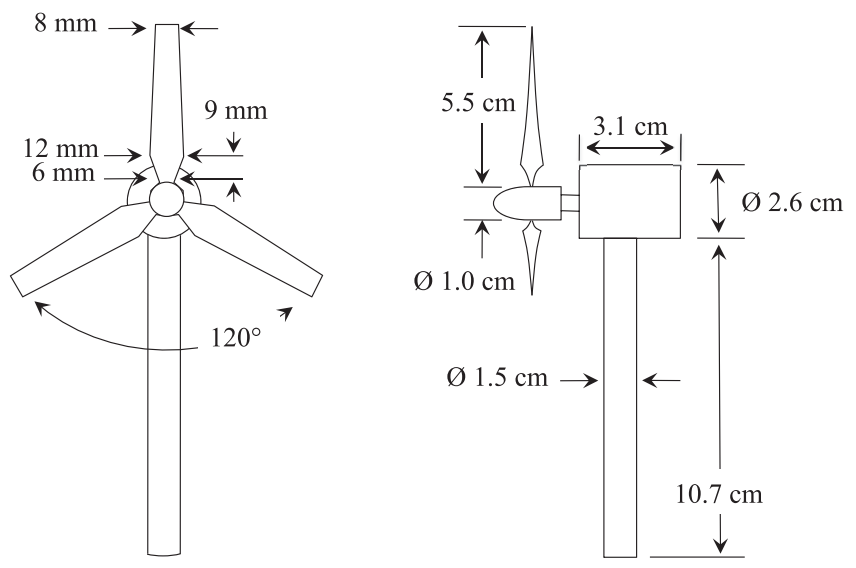

FIG. 2. Wind turbine model. 


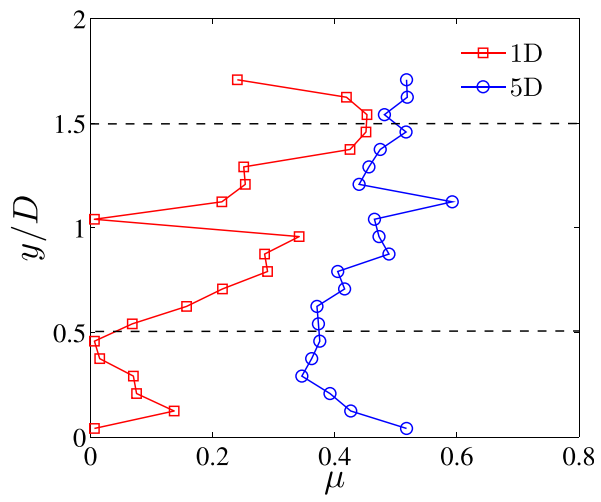

FIG. 3. Intermittency exponent $\mu$ as a function of vertical location at $1 D$ and $5 D$. The dashed lines represent the top and bottom tips of the wind turbine rotor.

whereas the correlation between the inertial and large scales generates the external intermittency. Vassilicos ${ }^{13}$ demonstrated that intermittency phenomenon violates the Galilean invariance on account of the combination between the large and small scales. Therefore, $\mu=0$ indicates non-existent interaction between integral scales and scales within the inertial subrange as confirmed by Katul et al. ${ }^{35}$ The top tip exhibits a maximum value of approximately 0.4 ; this being the location which demarcates the shear layer. As pointed out by Hamilton et al., ${ }^{32}$ it is at this vertical location, where the flow is described by lower wavenumbers or otherwise large scales, thus supporting the relatively increased intermittency exponent value.

Antonia et al., ${ }^{36}$ Chamber and Antonia, ${ }^{37}$ and Mahjoub et al. ${ }^{38}$ found $\mu=0.25 \pm 0.05$ for atmospheric shear flows (e.g., flow over a wheat field canopy), and a high Taylor-microscale based Reynolds number, $R e_{\lambda}$, jet flow. In these studies, Reynolds numbers range between 966 for the jet flow up to $10^{4}$ atmospheric surface layer. Further studies have revisited the intermittency exponent, thus finding $\mu=0.35$. This was shown by Anselmet $e t$ al. ${ }^{25}$ for the same data set utilized in Ref. 36 with a moderate Reynolds number, $R e_{\lambda}=515$ and 852, thus consequently emphasizing the impact of the Reynolds number on the $\mu$ value. Katul et al ${ }^{39}$ found $\mu=0.37$ for flow over a uniform sand dry lakebed where the $R e_{\lambda}$ extends between $5 \times 10^{3}$ and $3 \times 10^{4}$. Monin and Yaglom ${ }^{40}$ pointed out that $\mu$ in general ranges from 0.2 to 0.5 . Here, the intermittency exponent ranges from 0 to 0.6 ; bounding the range of previous values attained. Nevertheless, the magnitudes are affected due to the interaction between the flow and the wind turbine array, thus making $\mu$ dependent on the location within the wake of the array as well as the local Taylor-microscale based Reynolds number, $R e_{\lambda}$. For the data considered, $R e_{\lambda}$ ranges from a minimum of 300 for $1 D$ hub height location to 1600 at $5 D$ top tip.

In Figure 4, ESS scaling exponents of second, fourth, and fifth order structure functions at $1 D$ and $5 D$ are computed and compared with the K41, K62, SL, and Beta models to examine their affinity. The first and third order structure functions are disregarded in this study as a result of the negligible effect of intermittency on these moments as shown by Mahjoub. ${ }^{41}$ First, Figure 4(a) shows the ESS scaling exponent of the second order structure function at $1 D$, which decreases with increasing the wall-normal distance except at locations corresponding to the bottom tip, hub height, and top tip. Significant deviation from the various models is observed at all vertical locations with the exception of the aforementioned locations as well as near the wall. Indeed, immediately below the hub height, the ESS scaling manifests an agreement with K62 and SL. At the hub height, the scaling obtained via ESS coincides with K41, K62, and Beta. At the region above the hub height and above the canopy, ESS scaling exponents are located at the mid point among K41, K62, and SL models. In Figure 4(b), the scaling exponent of the second order of $5 D$ is relatively constant throughout the profile except in the near wall region. Conversely to $1 D$, ESS scaling exponents display a significant deviation from the other models along the wall-normal locations. This behavior also holds for the Beta model. However, 


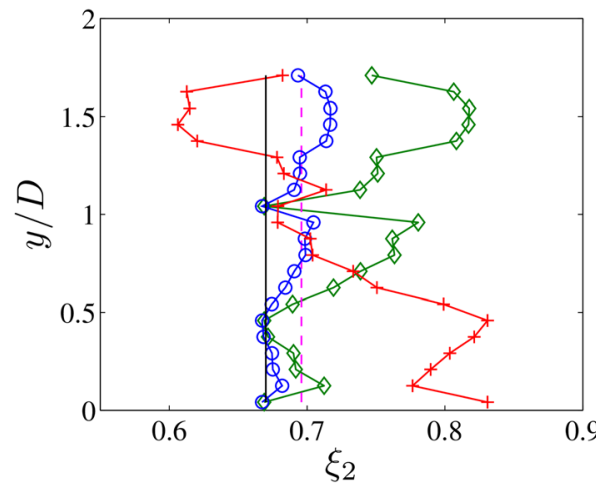

(a) $2^{\text {nd }}$ order- $1 D$.

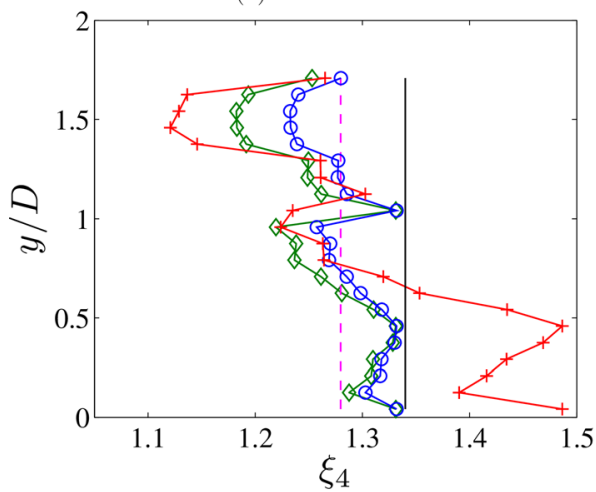

(c) $4^{\text {th }}$ order- $1 D$.

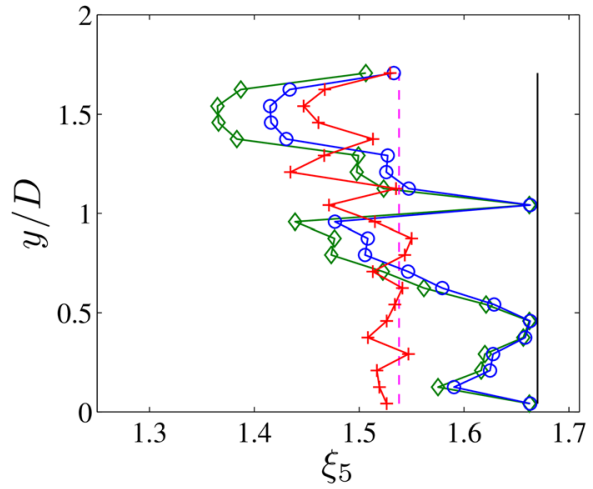

(e) $5^{\text {th }}$ order- $1 D$.

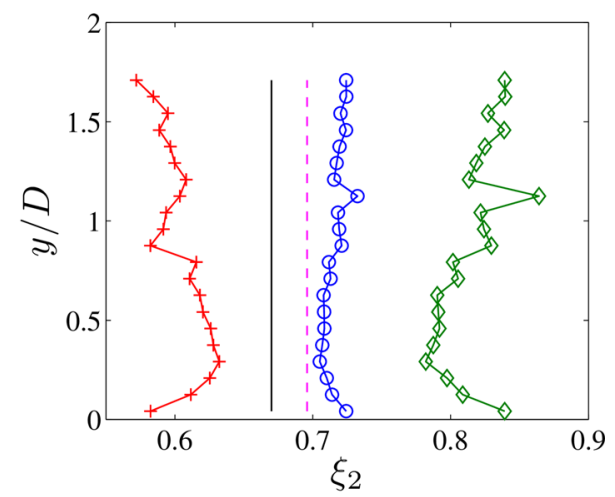

(b) $2^{\text {nd }}$ order- $5 \mathrm{D}$

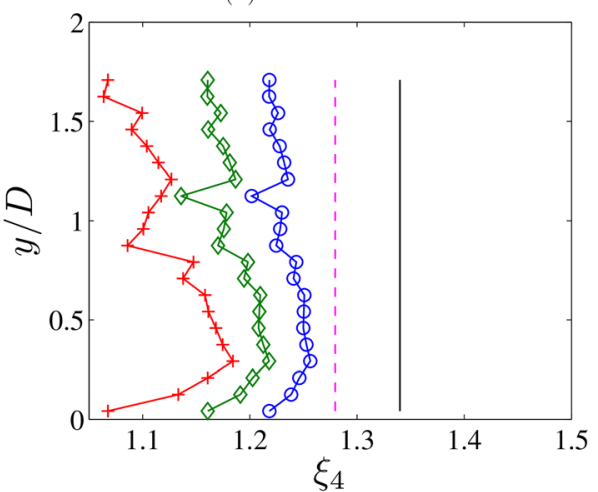

(d) $4^{\text {th }}$ order- $5 D$.

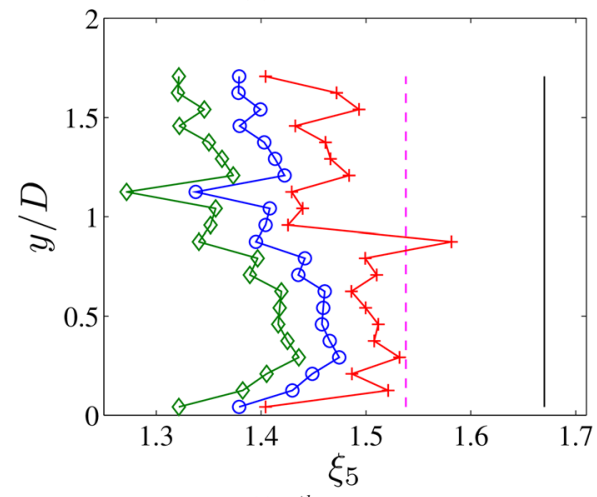

(f) $5^{\text {th }}$ order- $5 D$

FIG. 4. Scaling exponent of the second, fourth, and fifth orders compared with models in near and far wake regions. ESS $(+)$, Beta $(\diamond)$, SL $(---)$, K62 $(\bigcirc)$, and K41 (-). (a) 2nd order-1D, (b) 2nd order-5D, (c) 4th order-1D, (d) 4th order$5 D$, (e) 5 th order- $1 D$, and (f) 5 th order- $5 D$.

K62 model closely matches SL scaling exponents especially in the region between the hub height and bottom tip.

In Figures 4(c) and 4(d), the behavior of the ESS scaling exponent of the fourth order is rather similar to the second order at the same downstream location. Comparison amongst the models displays regions close to the hub height agreeing with K62 and SL as well as the hub height uniquely coinciding with the Beta model. Additionally, the region above the hub height and close to the top tip of $1 D$ is consistent with K62 and Beta in terms of their shape, although an offset exists, thus overpredicting the value of $\xi_{4}$. The highest measurement location away from the wall is consistent with all models except K41. In a like manner with the second order in the $5 D$ location, the fourth order structure function is overpredicted by all the models.

In Figure 4(e), ESS scaling exponents of the fifth order of $1 D$ slightly decrease with increasing wall-normal distance except at the regions above the bottom tip, hub height, and top 
tip. Once again, the models are not able to capture the values from the ESS with the exception of the top tip, where the Beta and K62 coincide. Finally, Figure 4(f) shows the scaling exponents of the fifth order for the far wake, where Beta and K62 are approximately consistent with the ESS scaling exponent profile in terms of shape with moderate deviation in terms of $\xi_{5}$ values. Furthermore, SL and K41 overpredict the results obtained for the ESS. Overall, the results of Figure 4 display that the intermittency events are pronounced in all three different orders of ESS scaling exponents and the deviation from the K41 is still pronounced even though the flow is 5 diameters downstream past the rotor.

To consider intermittency effects, the probability density function, pdf, is employed as its effects are observed in the tails as argued in Refs. 24, 25, and 42. The pdfs of the velocity increments, $\delta_{R}(u)$, for three scales, namely, $0.035,1.875 \times 10^{-3}$, and $0.9375 \times 10^{-3} \mathrm{~m}$, which correspond to $0.5 L, \lambda$, and $0.5 \lambda$, respectively, are shown logarithmically in Fig. 5. The results are limited to the hub height and top tip locations. These scales are chosen given their physical significance in the energy cascade, specifically in the inertial subrange. In Figure 5(a), representing the hub height location, the pdf becomes wider with an increase in scale, thus pointing to greater intermittency. At large scales, the pdfs collapse independent of the downstream position. In contrast, at smaller scales $\lambda$ and $0.5 \lambda$, the pdfs do not collapse.

In Figure 5(b), the pdfs at the same scales are shown, although now, at the top tip location. The large scale, $0.5 \mathrm{~L}$, tends to become more intermittent with increasing downstream distance where the tail is wider comparing with the pdf at $1 D$; thus, no collapse was observed between the distribution at the top tip of near- and far-wake. This vertical location physically coincides with the shear layer, where the flow above the canopy interacts with the flow below the top tip of wind turbine. Cal et al. ${ }^{30}$ highlighted that the wake of the wind turbine is recovered due to the flux from the flow above the canopy. Higher recovery takes place at the far-wake region, therefore the flow interaction at $5 D$ is much higher than $1 D$ which leads to an increased intermittency at $5 D$. Furthermore, the dependence with downstream position is not systematic since for the $\lambda$ scale, the pdfs become narrower with increasing downstream distance while at $0.5 \lambda$, the opposite occurs. This is due to the evolution of the scales as these move away from the wind turbine array. Nevertheless, it can be said that at the top tip and near the rotor, the pdfs are rather narrow, while in the far-wake the tails tend to be wider.

Based on the velocity increments, higher order statistics skewness, $S(R)=\frac{\left\langle\left(\delta_{R}(u)\right)^{3}\right\rangle}{\left\langle\left(\delta_{R}(u)\right)^{2}\right\rangle^{3 / 2}}$, and flatness, $F(R)=\frac{\left\langle\left\langle\delta_{R}(u)\right)^{4}\right\rangle}{\left\langle\left(\delta_{R}(u)\right)^{2}\right\rangle^{2}}$, are determined for hub height, bottom tip, and top tip regions at $1 D$ and $5 D$. In Figure 6(a), the skewness increases sharply with increasing spatial separation distance attaining a maximum value on scales, $R$, between 0.05 and $0.12 \mathrm{~m}$, and thereafter decreasing and converging to an approximately constant value. Following Katul et al., ${ }^{39,43}$ the structure of the flow is investigated through the constant skewness hypothesis which reveals the flow being locally anisotropic in the inertial subrange. At $1 D$, the skewness at hub height and bottom

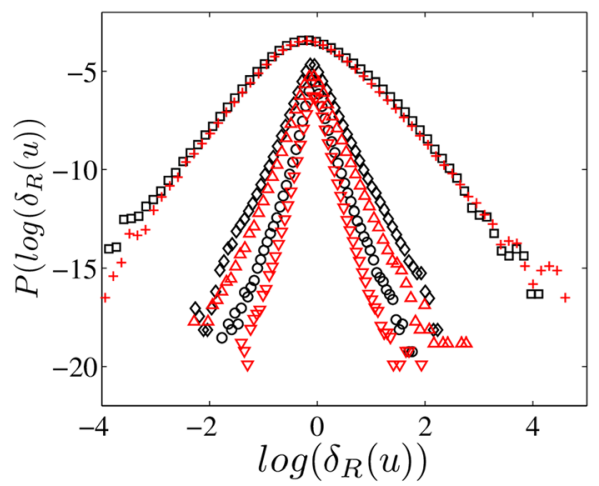

(a) Hub height.

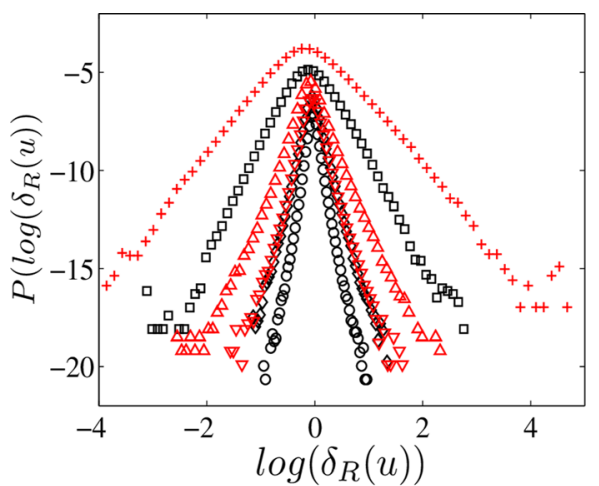

(b) Top tip.

FIG. 5. Probability density function of velocity increments for three scales at hub height and top tip for two downstream locations at (a) hub height and (b) top-tip. $0.5 L_{1 D}(\square), 0.5 L_{5 D}(+), \lambda_{1 D}(\diamond), \lambda_{5 D}(\nabla), 0.5 \lambda_{1 D}(\bigcirc), 0.5 \lambda_{5 D}(\triangle)$. 


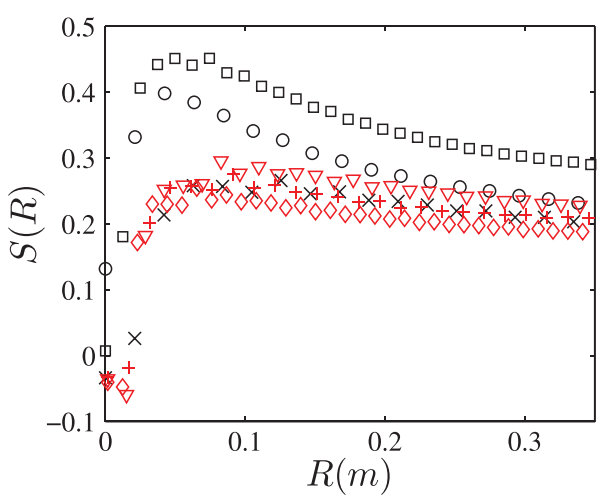

(a) Skewness.

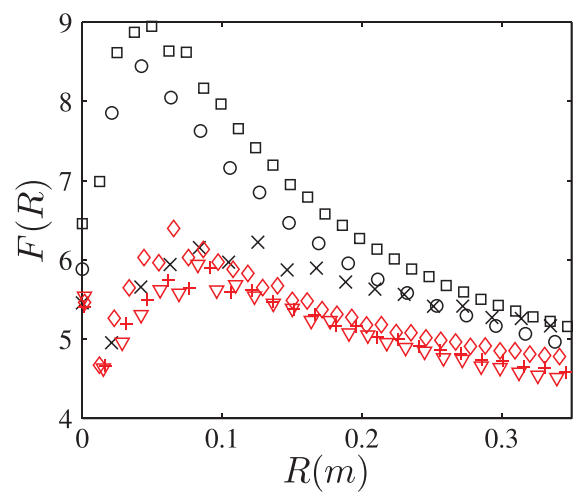

(b) Flatness.

FIG. 6. Skewness (a) and flatness (b) for hub height and tip regions at $1 D$ and $5 D$. Bottom tip- $1 D(\bigcirc)$, hub height- $1 D$ $(\square)$, top tip $-1 D(\times)$, bottom tip $-5 D(\nabla)$, hub height $-5 D(+)$, and top tip $-5 D(\diamond)$.

tip decreases at a more rapid rate than top tip, and the highest constant skewness displayed at the hub height is about 0.28 , comparing with other regions having approximately $S(R)$ between 0.18 and 0.22 . The range of the skewness agrees well within the range presented by Townsend $^{44}$ and Monin and Yaglom, ${ }^{40}$ where the suggested skewness ranges are $0.22-0.3$ and 0.2-0.45 for an atmospheric surface layer, respectively.

In Figure 6(b), the flatness strongly increases with increasing $R$ until reaching a maxima between $R=0.05-0.12 \mathrm{~m}$. Beyond these $\mathrm{R}$ values, the flatness monotonically decreases until attaining an approximately constant value approximately between 4 and 5 . Flatness of the $1 D$ location steeply decreases with increasing scales, and shows large deviation from a kurtosis of 3 corresponding to a normal distribution. With moving downstream, the flatness shows a significant variation of the maximum value compared with $1 D$ locations. Thus, the profiles of the flatness show a slight decrease with scales and deviation from Gaussian distribution. A comparison of the two locations, $1 D$ and $5 D$, reveals that the maximum flatness associated with the range of scales, $\mathrm{R}$, occurs at the hub height and bottom tip at the location $1 D$. Conversely, the maximum flatness at far-wake region occurs at the top tip.

\section{CONCLUSIONS}

Intermittency phenomenon in turbulence flow is the local fluctuations in energy dissipation or velocity increments. This phenomenon can be identified with the use of scaling exponents of different moments, associating with the separation scales between two points. Wind tunnel experiments were performed to study intermittency phenomena in the near, $1 D$, and far-wake regions, $5 D$, of a wind turbine array placed in a boundary layer. Scaling exponents of the sixth order structure functions are used to compute the intermittency exponents that are generally found extending between 0 and 0.6 ; higher values coinciding with the far-wake location. ESS scaling exponents of second, fourth and fifth orders are calculated. The results show that the scaling exponents of $5 D$ are approximately constant whereas at $1 D$ these significantly vary as a function of height especially at the top tip, bottom tip and hub height locations.

Near-wake regions show that the second order of ESS scaling exponents is consistent with SL and K62 at the region located below the hub height and above the bottom tip. A slightly deviation from K41, Beta, and K62 occurred at the hub height. The largest deviation from the other models happens at the bottom and top tip. The fourth order of ESS scaling exponent shows a significant deviation at the region extending between the wall and above the bottom tip. The Beta model manifests consistence with ESS scaling exponents at the region below the hub height and the mid region between the hub height and top tip. Thus, the hub height and top tip show a significant deviation from the other models. The fifth order of ESS scaling exponents is consistent with SL model above the bottom tip and above the hub height. The highest wall-normal region displays agreement with SL and K62 models. In the far-wake region, ESS 
exponents of fourth and fifth orders are consistent in terms of their trend with Beta and K62 models, although the values are overpredicted. The tails of the pdfs reveal that the intermittency effect at $5 D$ is higher than $1 D$. Flatness and skewness reach the maximum values at the same range of $R$. The constant flatness and skewness ranges are 4-5 and 0.18-0.22, respectively.

The nature of the flow within an array of wind turbines has been characterized in the context of structure functions and intermittency. This study provides insight on intermittency events in the near- and far-wake past a wind turbine array as intermittency effects are observed in both locations. Future research may concentrate on the investigation of intermittency based on wind farm siting variations.

\section{ACKNOWLEDGMENTS}

The authors thank the reviewers whose constructive comments helped to improve this work. This work is in part funded by the National Science Foundation (Grant No. CBET-1034581).

${ }^{1}$ G. W. E. Council, GWEC, November (2012).

${ }^{2}$ W. Zhang, C. D. Markfort, and F. Porté-Agel, Exp. Fluids 52, 1219 (2012).

${ }^{3}$ N. Ali, A. Aseyev, J. McCraney, V. Vuppuluri, O. Abbass, T. Al Jubaree, M. Melius, and R. B. Cal, Bull. Am. Phys. Soc. 59, 423 (2014).

${ }^{4}$ L. Vermeer, J. N. Sørensen, and A. Crespo, Prog. Aerosp. Sci. 39, 467 (2003).

${ }^{5}$ M. Calaf, C. Meneveau, and J. Meyers, Phys. Fluids 22, 015110 (2010).

${ }^{6}$ N. Hamilton, M. Melius, and R. B. Cal, Wind Energy 18(2), 277-295 (2014).

${ }^{7}$ M. Chamecki and N. Dias, Q. J. R. Meteorol. Soc. 130, 2733 (2004).

${ }^{8}$ V. I. Tatarskii, Wave Propagation in Turbulent Medium, translated by R. A. Silverman (McGraw-Hill, 1961$)$, Vol. 1.

${ }^{9}$ A. N. Kolmogorov, Proc.: Math. Phys. Sci. 32, 15 (1941).

${ }^{10}$ S. B. Pope, Turbulent Flows (Cambridge University Press, 2000).

${ }^{11}$ C. Meneveau and K. Sreenivasan, J. Fluid Mech. 224, 429 (1991).

${ }^{12}$ A. N. Kolmogorov, J. Fluid Mech. 13, 82 (1962).

${ }^{13}$ J. C. Vassilicos, Intermittency in Turbulent Flows (Cambridge University Press, 2001).

${ }^{14}$ U. Frisch, P.-L. Sulem, and M. Nelkin, J. Fluid Mech. 87, 719 (1978).

${ }^{15}$ O. N. Boratav and R. B. Pelz, Phys. Fluids 9, 1400 (1997).

${ }^{16}$ J. Gao, Y. Cao, W.-W. Tung, and J. Hu, Multiscale Analysis of Complex Time Series: Integration of Chaos and Random Fractal Theory, and Beyond (John Wiley \& Sons, 2007).

${ }^{17}$ R. Benzi, S. Ciliberto, R. Tripiccione, C. Baudet, F. Massaioli, and S. Succi, Phys. Rev. E 48, R29 (1993).

${ }^{18}$ A. Babiano, B. Dubrulle, and P. Frick, Phys. Rev. E 55, 2693 (1997).

${ }^{19}$ M. Briscolini, P. Santangelo, S. Succi, and R. Benzi, Phys. Rev. E 50, R1745 (1994).

${ }^{20}$ A. E. Arnéodo, C. Baudet, F. Belin, R. Benzi, B. Castaing, B. Chabaud, R. Chavarria, S. Ciliberto, R. Camussi, F. Chilla et al., Europhys. Lett. 34, 411 (1996).

${ }^{21}$ E. Gaudin, B. Protas, S. Goujon-Durand, J. Wojciechowski, and J. Wesfreid, Phys. Rev. E 57, R9 (1998).

${ }^{22}$ Z.-S. She and E. Leveque, Phys. Rev. Lett. 72, 336 (1994).

${ }^{23}$ B. Dubrulle, Phys. Rev. Lett. 73, 959 (1994).

${ }^{24}$ J. M. Vindel, C. Yage, and J. M. Redondo, Nonlinear Processes Geophys. 15, 915 (2008).

${ }^{25}$ F. Anselmet, Y. Gagne, E. J. Hopfinger, and R. A. Antonia, J. Fluid Mech. 140, 63 (1984).

${ }^{26}$ A. Vincent and M. Meneguzzi, J. Fluid Mech. 225, 1 (1991).

${ }^{27}$ P. D. Ditlevsen and I. Mogensen, Phys. Rev. E 53, 4785 (1996).

${ }^{28}$ P. Milan, M. Wächter, and J. Peinke, Phys. Rev. Lett. 110, 138701 (2013).

${ }^{29}$ T. Mücke, D. Kleinhans, and J. Peinke, Wind Energy 14, 301 (2011).

${ }^{30}$ R. B. Cal, J. Lebrón, L. Castillo, H. S. Kang, and C. Meneveau, J. Renewable Sustainable Energy 2, 013106 (2010).

${ }^{31}$ M. S. Melius, M. Tutkun, and R. B. Cal, J. Renewable Sustainable Energy 6, 023121 (2014).

${ }^{32}$ N. Hamilton, H. S. Kang, C. Meneveau, and R. B. Cal, J. Renewable Sustainable Energy 4, 063105 (2012).

${ }^{33}$ F. Böttcher, S. Barth, and J. Peinke, Stochastic Environ. Res. Risk Assess. 21, 299 (2007).

${ }^{34}$ V. Kuznetsov, A. Praskovsky, and V. Sabelnikov, J. Fluid Mech. 243, 595 (1992).

${ }^{35}$ G. G. Katul, M. B. Parlange, and C. R. Chu, Phys. Fluids 6, 2480 (1994).

${ }^{36}$ R. Antonia, N. Phan-Thien, and B. Satyaprakash, Phys. Fluids 24, 554 (1981).

${ }^{37}$ A. Chambers and R. Antonia, Boundary-Layer Meteorol. 28, 343 (1984).

${ }^{38}$ O. B. Mahjoub, J. M. Redondo, and A. Babiano, Appl. Sci. Res. 59, 299 (1997).

${ }^{39}$ G. G. Katul, M. B. Parlange, J. D. Albertson, and C. R. Chu, Boundary-Layer Meteorol. 72, 123 (1995).

${ }^{40}$ A. Monin and A. Yaglom, Statistical Fluid Mechanics (MIT Press, 1975), Vol. II.

${ }^{41}$ O. B. Mahjoub, Ph.D. thesis, Universitat Politècnica de Catalunya, 2001.

${ }^{42}$ R. A. Antonia, A. J. Chambers, and B. R. Satyaprakash, Boundary-Layer Meteorol. 21, 159 (1981).

${ }^{43}$ G. Katul, C.-I. Hsieh, and J. Sigmon, Boundary-Layer Meteorol. 82, 49 (1997).

${ }^{44}$ A. A. Townsend, The Structure of Turbulent Shear Flow (Cambridge University Press, 1980). 